Ann. Génét. Sél. anim., 1982, 14 (3), 399-414

Revue bibliographique

\title{
L'héritabilité des performances chez le Trotteur (1) Une revue bibliographique
}

\author{
B. LANGLOIS \\ I.N.R.A., Station de Génétique quantitative et appliquée \\ Centre de Recherches zootechniques, 78350 Jouy-en-Josas
}

\begin{abstract}
Résumé
La bibliographie des travaux de génétique quantitative sur le Trotteur permet de conclure à une héritabilité moyenne des performances réalisées en course $\left(0,20<\mathrm{h}^{2}<0,30\right)$.

Les critères disponibles issus des résultats dans une épreuve traduisent plus le niveau de la course dans laquelle un cheval est capable de figurer que ses véritables possibilités dans l'absolu. Il y a de ce point de vue peu de différences entre les temps chronométrés et les variables issues des gains. Dans un cas, le niveau de la course est exprimé par la vitesse moyenne de l'épreuve, dans l'autre par sa dotation.

L'influence des effets systématiques de l'âge, du sexe, de l'année de la performance, de l'hippodrome, du type de départ, du mode attelé ou monté, etc., est passée en revue, comme les problèmes statistiques particuliers liés aux distributions des variables autres que les temps chronométrés.

L'estimation de la valeur génétique des reproducteurs est ensuite abordée sous l'angle du contrôle individuel et du contrôle de la descendance.

On conclut sur l'évolution possible des travaux de recherche dans ce domaine et leur éventuelle application.
\end{abstract}

\section{Introduction}

Comme le souligne Minkema (1975 a), l'intérêt des généticiens pour les performances des trotteurs est ancien puisque GaLton (1898), l'un des fondateurs de la génétique quantitative, écrivait à la fin du siècle dernier :

«Il y a dans les registres de l'association du trotteur américain un matériel des plus intéressants pour la recherche des lois de l'hérédité $» .$.

Cet intérêt ne s'est cependant concrétisé que tardivement et essentiellement durant ces dix dernières années : Minkema (1978) surtout puis Hintz (1980) ont commencé à faire le point de nos connaissances sur le sujet. Il nous a paru utile de compléter et d'approfondir ce travail de mise au point.

(1) Conférence soutenue le 10 mars 1982 à l'occasion de la $8^{\text {e }}$ Journée d'étude sur le cheval organisée par le C.E.R.E.O.P.A., 16, rue Claude-Bernard, Paris. 


\section{Les mesures des performances}

Pour toutes les études, on constate que la structure élémentaire des informations concernant les performances d'un trotteur est toujours la même. Elle exprime le résultat d'un cheval dans une course par :

- un chronométrage,

- un gain,

- une place.

Toutefois, la prise en considération de l'ensemble de ces informations pour les études génétiques conduit à manipuler des fichiers gigantesques. A titre d'exemple, en France, 10000 chevaux environ participent chacun à une dizaine de courses en moyenne par an, soit un volume de 100000 enregistrements pour une seule année. Or, l'intervalle de génération chez le trotteur a été estimé à environ 12 ans (de Ricister, 1977), ce qui, combiné à la longueur de la carrière de course, oblige dans les études génétiques à prendre en compte un grand nombre d'années pour avoir une bonne vue d'ensemble.

On comprend donc aisément la nécessité qu'ont ressentie à la fois les utilisateurs et les chercheurs de recourir à des critères synthétiques qui peuvent être élaborés au niveau de l'année de compétition ou bien de l'ensemble de la carrière. Ces critères sont en général :

- des temps moyens,

- des records,

- des sommes de gains,

- des nombres de courses, de classements ou de victoires.

On peut d'ailleurs en imaginer un nombre très important en combinant les informations élémentaires, mais les tro:s grandes catégories peuvent en général toujours ĉtre distinguées soit :

Les critères issus du chronomètre.

Les critères issus des gains.

Les critères issus de la place.

Les premiers, dont la distribution est très proche de la normalité, peuvent être traités tels quels et, soumis à l'analyse statistique classique. Quelques auteurs (MinkEMA, 1975 ; ÁRNASON et al., 1982 a) ont cependant tenté, sans grand succès dailleurs, d' « améliorer» encore la normalité de la distribution.

Les critères de gain en revanche ont des histogrammes qui traduisent de forts ćcarts à la normalité. Beaucoup d'auteurs leur ont donc appliqué des transformations. Etant donné l'étalement des distributions de gains vers les valeurs fortes, on a surtout cherché à ramener vers la moyenne les valeurs extrêmes par l'utilisation de transformations dites "stabilisatrices de variance». Alors que la racine carrée semble suffire pour normaliser la distribution des gains des trotteurs hollandais (MINKEMA, 1975, 1982) et finlandais (OJALA \& VAN VLECK, 1981) qui sont sans doute assez homo- 
gènes, il faut recourir à la racine cubique (DEscops, 1976) voire à la racine quatrième (ÁRNASON et al., 1982 a) pour obtenir le même résultat avec des gains plus étalés. A notre sens, la transformation logarithmique présente en la matière, une certaine universalité car elle correspond à une réalité. En effet, l'attribution des prix aux chevaux en fonction de leur classement se fait selon des règles de type exponentiel (pourcentage à peu près constant de décroissance du gain en fonction du classement). L'application des transformations logarithmiques tend donc à stabiliser l'estimation de l'écart de place quels que soient le niveau de la course et le classement (LANGLoIs, 1982). C'est ce qui lui confère son efficacité générale dans le traitement des variables issues des gains : une bonne illustration en est donnée par THERY (1981). Notons encore que, dans certains cas, les gains à un âge donné ou sur une gamme de distances données sont exprimés en pourcentage des gains totaux. C'est le cas des critères de précocité, d'aptitude aux courtes distances et de tenue proposés par MinKEMA (1975). Il s'agit respectivement du pourcentage $\mathrm{x}$ des gains obtenus sur des distances comprises entre 1600 et $2000 \mathrm{~m}$, supérieures ou égales à $2400 \mathrm{~m}$ et de la proportion des gains totaux de la carrière obtenus à l'âge de 2 et 3 ans. Lorsque les valeurs de $\mathrm{x}$ sont trop proches de 0 ou de 1 , la distribution ne peut pas non plus être supposée normale et le recours à des transformations du type $\operatorname{Logit}[\log (x+0,5)-\log (100,5-x)]$ ou de type logarithmique $[\log (x+0,5)]$ a été proposé.

Le remplacement des transformations stabilisatrices de variance classiques par des fonctions dites «logistiques» (fonctions qui permettent de passer de l'intervalle $-1,+1$ à $-\infty,+\infty)$ dans le cas de l'étude de pourcentages, est également caractéristique des variables issues de la place.

Pour le traitement des variables, pourcentage de victoires, pourcentage de classements et pourcentage de disqualification, OJALA \& VAN VLECK (1981) proposent en effet à côté de la racine carrée, l'utilisation des fonctions Arcsinus et Logit. D'autres auteurs, malgré les écarts importants à la normalité de ce type de variable, ne se préoccupent pas des problèmes de distribution comme d'ailleurs dans le traitement des variables de gain (Linner \& OSterkorn, 1974 ; RönNingen, 1975 ; KATONA, 1979). Cela dans certains cas diminue en partie la portée de leurs résultats.

\section{Les principaux facteurs de milieu influençant les performances}

\section{A. L'élevage d'origine et l'entraînement}

Ces facteurs sont très certainement de nature à influer sur les performances. Signalons toutefois que la longueur de la carrière de compétition chez le trotteur peut permettre avec le temps de compenser d'éventuels retards de développement ou certaines phases de méforme. Cette possibilité distingue très nettement la discipline du trot de celle du galop vis-à-vis de ce type d'effets.

En théorie, connaissant l'éleveur, l'entraîneur et le driver d'un cheval, il est possible statistiquement d'éliminer leur influence sur les performances des chevaux. Toutefois, en pratique, le nombre d'éleveurs, d'entraîneurs et de drivers à considérer, la taille des fichiers nécessaires, l'importance des calculs numériques à réaliser, sont des freins importants pour mener à bien de telles opérations. Dans la littérature, la 
seule tentative de ce genre a été conduite par Hintz \& VAN VLECK (1978) : les différences entre drivers expliquent 11 p. 100 de la variance des temps réalisés par les ambleurs américains tout en ne concluant pas définitivement sur la signification de cet effet.

\section{B. L'année de la performance ou l'année de naissance du cheval}

Ce facteur intervient bien évidemment sur les sommes gagnées du fait de l'évolution des dotations mais également sur les temps en raison des progrès réalisés au cours des années dans la fabrication des sulkys, l'amélioration des méthodes d'entraînement et surtout dans la qualité des pistes. Minkema (1975) travaillant sur des données étalées sur de longues périodes a ressenti cette nécessité. Il a donc exprimé chaque record individuel en écart à une courbe de référence établie par ajustement des moyennes annuelles. Il souligne cependant que cette correction a l'inconvénient de corriger non seulement pour les effets du milieu mais aussi, ce qui n'est pas souhaitable, pour les progrès d'origine génétique. Dans une publication ultérieure (Minkema, 1981), il estime qu'environ 80 p. 100 des progrès observés chez le trotteur hollandais sont probablement d'origine génétique ce qui remet en question l'opportunité de telles corrections sur les temps.

Leur nécessité apparaît en revanche clairement pour le gain, ne serait-ce qu'en raison de l'inflation. La méthode traditionnelle d'actualisation financière (MINKEMA, 1975 ; DescoQs, 1976) consiste à multiplier le gain obtenu par un cheval durant l'année i par le rapport de la moyenne de l'année de référence $\mathrm{i}_{\mathrm{o}}$ à la moyenne de l'année i. Il y a lieu dans ce cas de calculer des coefficients de correction pour chaque type de variable (gain annuel, gains totaux, gain moyen par départ...) car les fluctuations de la dotation, du nombre de concurrents et du nombre de courses par cheval peuvent parfois suivre des évolutions différentes. L'avantage de ce type de correction est de pouvoir analyser les variables brutes avant transformation sans avoir besoin de disposer des performances des chevaux contemporains (seule la moyenne de l'année pour la variable est nécessaire).

Dans d'autres études (RöNNINGEN, 1975), cette correction est tentée de manière additive. On estime ainsi pour chaque année un facteur à ajouter ou à retrancher, par l'analyse de la variance. Cela suppose que l'on dispose de la totalité du matériel pour l'année estimée de façon à éviter les biais d'échantillonnage liés à une sélection des données. Il faut également travailler sur les variables transformées pour que les conditions de normalité soient remplies.

\section{L'âge}

Tous les auteurs s'accordent pour souligner l'importance de l'effet de l'âge qu'il est impératif de prendre en compte.

Les performances s'améliorent avec l'âge, jusqu'à ce que le vieillissement intervenant, elles finissent de nouveau par diminuer. Ces processus se traduisent sur toutes les variables (temps, nombre de sorties), mais n'ont pû être analysés correctement que dans leur phase ascendante, les chevaux étant progressivement retirés des compétitions à mesure que leurs performances décroissent. 


\author{
D. Le sexe
}

La supériorité des mâles sur les femelles s'observe pour les temps (LINNER, 1973 ; Linner \& Osterkorn, 1974 ; Katona \& Osterkorn, 1977 ; Thery, 1981 ; Ojala, 1982), les records (Minkema, 1975 ; RönNingen, 1975), les gains (Minkema, 1975 ; RönNingen, 1975 ; THERY, 1981) et le nombre de victoires ou de classements (RöNNINGEN, 1975).

Les hongres ne sont en général pas distingués des mâles, ou bien on évite de corriger pour ce facteur, la castration n'intervenant pas indépendamment de la qualité des chevaux concernés. En effet on hésite à castrer de bons chevaux et lorsqu'on le fait c'est uniquement pour obtenir des performances plus régulières, quand leur caractère les rend difficiles à utiliser.

\title{
E. L'hippodrome
}

Ce facteur n'influence pas les gains et les critères de classement dans les pays où il y a peu de lieux de courses et où de ce fait il ne s'établit pas de hiérarchie entre eux. Si cela peut être considéré pour certains pays européens, c'est en revanche loin d'être le cas en France où le niveau des courses est très différent d'un hippodrome à l'autre (THERY, 1981) et se traduit par d'importantes variations de dotation et de nombre de courses.

La plus ou moins grande rapidité des hippodromes constitue cependant un facteur que certains auteurs jugent nécessaire de corriger (LiNNER, 1973 ; LiNNER \& Osterkorn, 1974 ; Katona \& Osterkorn, 1977 ; Thery, 1981). D'autres (Minkema, 1975 ) estiment que c'est une correction délicate, les hippodromes étant souvent modifiés et le plus souvent rendus plus rapides, on ne peut se fier aux estimations obtenues à un instant donné. Il pense par ailleurs que les chevaux courent sur de nombreux hippodromes et qu'ils ont de ce fait la possibilité un jour ou l'autre de s'exprimer dans les meilleures conditions. C'est un argument également utilisé pour se dispenser de corriger pour l'état du terrain qualifié de rapide, bon, moyen, lourd ou enneigé qui peut entraîner des variations de plusieurs secondes que HiNTz \& VAN Velck, 1978 ; LINNER \& OSTERKORN et KATONA \& OSTERKORN, 1977, se sont attachés à éliminer. Signalons également les différences entre pistes en herbe et pistes en cendrée (THERY, 1981) qui engendrent des variations de même ordre.

\section{F. Les conditions particulières de la course}

Certains auteurs (CSAPó, 1968, Linner \& OSTERKorn, 1974 ; KATONA \& OsTerKORN, 1977) se sont attachés à estimer l'effet de la température ou de la saison sur les temps de courses. Dans le même ordre d'idées, l'humidité de l'air, la direction et la vitesse du vent, le fait que la course ait lieu le jour ou en nocturne influencent les temps réalisés par les chevaux. L'incidence de ces facteurs semblent toutefois assez faible et reste à ce jour encore difficile à saisir étant donnés les liens étroits qui existent entre l'état du terrain, la température, l'humidité et l'éclairement (nocturne ou diurne).

La distance, les conditions de départ, le fait que la course soit montée ou attelée ont en revanche une influence supérieure et mieux caractérisée. En ce qui concerne 
le premier facteur, on considère que la réduction kilométrique (THERY, 1981) croît d'environ $1 / 10$ de seconde lorsque la distance s'allonge de $100 \mathrm{~m}$, ce qui n'est pas négligeable. Le deuxième groupe de facteurs englobe la position de départ, dont l'influence a été soulignée par HinTz \& VAN VLECK (1978), et le mode de départ. On reconnaît le plus souvent un avantage au départ à l'autostart sur le départ aux élastiques, avantage confirmé par LINNER \& OsTERKORN (1974), non vérifié par KatONA \& OsterkoRN (1977), et estimé à 1 seconde sur d'importants effectifs en France (Thery, 1981). Un écart équivalent a d'ailleurs été trouvé entre l'attelé et le monté.

Il nous semble toutefois que les conditions particulières de la course qui influencent le plus les performances ne tiennent pas aux effets systématiques décrits plus haut. Le train de la course, sous la dépendance de facteurs tactiques mais surtout étroitement lié à la qualité des concurrents qui y participent, nous paraît avoir en fait beaucoup plus d'importance. L'influence du niveau de dotation sur les temps réalisés soulignée par LINNER (1973) et HINTZ \& VAN VLECK (1978) en est une illustration. Il est cependant difficile et sans doute peu souhaitable de corriger pour ce type d'influences essentiellement aléatoires et qui fixent en fait le niveau de l'épreuve à travers lequel les chevaux expriment leur aptitude. Certains auteurs (FERGUSON \& Harvey, 1982; Tolley \& NotTer, 1982) ne sont pas de cet avis et proposent de régresser chaque chronométrage en fonction du temps du premier pour aboutir à un résultat corrigé pour le train de la course.

\section{Héritabilité des critères de performances}

Les tableaux 1,2 et 3 résument les nombreuses estimations publiées concernant les critères chronométriques, les gains et les variables qui découlent du classement. Quand les auteurs estiment l'héritabilité par plusieurs méthodes et ne fournissent pas d'estimation combinée, nous avons procédé au calcul de cette estimation combinée en fonction des effectifs fournis de façon à regrouper le plus possible les éléments disponibles.

Les études les plus nombreuses concernent les temps (tabl. 1) dont 20 à 30 p. 100 de la variation peut ètre considérée comme d'origine génétique. On peut remarquer que paradoxalement il semble que le fait de passer à des critères synthétiques au niveau de l'année ou de la carrière ne s'accompagne pas d'une augmentation sensible de l'héritabilité. Ce passage se fait donc le plus souvent avec trop de perte d'information puisqu'on ne gagne pas en précision malgré un nombre beaucoup plus important de performances considérées par animal. Ceci est à rapprocher des observations de KATONA \& OSTERKORN (1977) et de Katona (1979) qui constatent respectivement qu'un seul chronométrage a une corrélation de 0,70 et 0,78 avec la moyenne annuelle du cheval. Ils en concluent que trois ou quatre réductions kilométriques par an sont suffisantes pour apprécier les performances d'un cheval, les informations supplémentaires n'apportant plus grand chose. La répétabilité $r=0,52 \mathrm{du}$ temps de course trouvée pour le trotteur français (THERY, 1981) confirme cette option, de même que celles avancées pour des effectifs plus restreints sur le trotteur finlandais par OJala \& VAN Vleck (1981) : 0,61<r $<0,74$ selon l'âge.

Entre moyennes annuelles consécutives, les corrélations croissent de 0,63 à 0,79 de 2 à 5 ans et restent ensuite stables (KATONA \& OSTERKORN, 1977). Les chiffres 


\section{TABLEAU 1}

Récapitulatif des estimations de l'héritabilité et de la répétabilité de la vitesse au trot. (les valeurs marquées d'un astérisque ${ }^{(*)}$ sont des moyennes calculées par nos soins).

Heritability and repeatability of trotting speed.

(Values with an asterisk (*) are calculated means).

\begin{tabular}{|c|c|c|c|}
\hline Critère & Référence & Héritabilité & Répétabilité \\
\hline $\begin{array}{l}\text { Test de vitesse au trot pour } \\
\text { chevaux de trait } \ldots \ldots \ldots \text {. }\end{array}$ & $\begin{array}{l}\text { LONKA }(1946) \\
\text { VAINIKAINEN }(1946) \\
\text { VARO }(1965)\end{array}$ & $\begin{aligned} \mathrm{h}^{2} & =0,62 \\
{ }^{*} \mathrm{~h}^{2} & =0,50 \\
\mathrm{~h}^{2} & =0,43\end{aligned}$ & \\
\hline Temps dans une course & $\begin{array}{l}\text { Hintz \& VAN Vleck (1978) } \\
\text { OJALA \& VAN VleCK (1981) } \\
\text { THERY (1981) } \\
\text { TOLLEY \& NotTER (1982) }\end{array}$ & $\begin{aligned}{ }^{*} \mathrm{~h}^{2} & =0,31 \\
\mathrm{~h}^{2} & =0,23 \\
\mathrm{~h}^{2} & =0,29\end{aligned}$ & $\begin{array}{rl}* & r=0,32 \\
* & =0,68 \\
r & =0,52 \\
r & =0,44\end{array}$ \\
\hline Moyenne annuelle & $\begin{array}{l}\text { SCHWARK \& FREUND }(1974,1976) \\
\text { LINNER \& OSTERKORN }(1974) \\
\text { KATONA \& OSTERKORN }(1977) \\
\text { KATONA (1979) } \\
\text { OJALA \& VAN VLECK }(1981)\end{array}$ & $\begin{aligned} \mathrm{h}^{2} & =0,21 \\
\mathrm{~h}^{2} & =0,21 \\
\mathrm{~h}^{2} & =0,20 \\
\mathrm{~h}^{2} & =0,30 \\
* \mathrm{~h}^{2} & =0,23\end{aligned}$ & $\begin{aligned} r & =0,45 \\
* r & =0,77 \\
r & =0,75 \\
r & =0,76\end{aligned}$ \\
\hline Record annuel & $\begin{array}{l}\text { SCHWARK \& FREUND }(1974,1976) \\
\text { WRANKMORE }(1976) \\
\text { GEORGESCU et a!. (1979) } \\
\text { KATONA (1979) } \\
\text { OJALA \& VAN VLECK }(198.1) \\
\text { OJALA (1982) }\end{array}$ & $\begin{aligned} * \mathrm{~h}^{2} & =0,18 \\
\mathrm{~h}^{2} & =0,38 \\
{ }^{*} \mathrm{~h}^{2} & =0,29 \\
{ }^{*} \mathrm{~h}^{2} & =0,18 \\
{ }^{*} \mathrm{~h}^{2} & =0,43 \\
{ }^{*} \mathrm{~h}^{2} & =0,08\end{aligned}$ & $\begin{array}{l}r=0,34 \\
r=0,61 \\
r=0,69 \\
r=0,55\end{array}$ \\
\hline Moins bon temps annuel .. & Ojala \& Van Vleck (1981) & ${ }^{*} \mathrm{~h}^{2}=0,35$ & $r=0,53$ \\
\hline Record absolu & $\begin{array}{l}\text { ÓCSAG \& Tóth (1959) } \\
\text { GoPKA (1970-1971 a, 1971 b) } \\
\text { KALMYKOV (1973 a, 1973 b, 1975) } \\
\text { SCHWARK \& FREUND }(1974,1976) \\
\text { MINKEMA (1975) } \\
\text { RöNNINGEN (1975) } \\
\text { DESCOOS (1976) } \\
\text { DUŠEK (1979) } \\
\text { HINTZ (1980) } \\
\text { ÁRNASON et al. (1982 a) }\end{array}$ & $\begin{aligned} \mathrm{h}^{2} & =0, \mathrm{ch} \\
\mathrm{k}^{2} & =0,42 \\
* \mathrm{~h}^{2} & =0,34 \\
\mathrm{~h}^{2} & =0,21 \\
\mathrm{~h}^{2} & =0,36 \\
\mathrm{~h}^{2} & =0,12 \\
\mathrm{~h}^{2} & =0,21 \\
\mathrm{~h}^{2} & =0,23 \\
\mathrm{~h}^{2} & =0,28\end{aligned}$ & $\begin{aligned} r & =0,45 \\
r & =0,34 \\
{ }^{*} r & =0,45\end{aligned}$ \\
\hline
\end{tabular}




\section{TABleau 2}

Récapitulatif des estimations de l'héritabilité et de la répétabilité des critères de gains. (les valeurs marquées d'un astérisque (*) sont des moyennes calculées par nos soins).

Heritability and repeatability of earning criteria.

(Values with an asterisk (*) are calculated means).

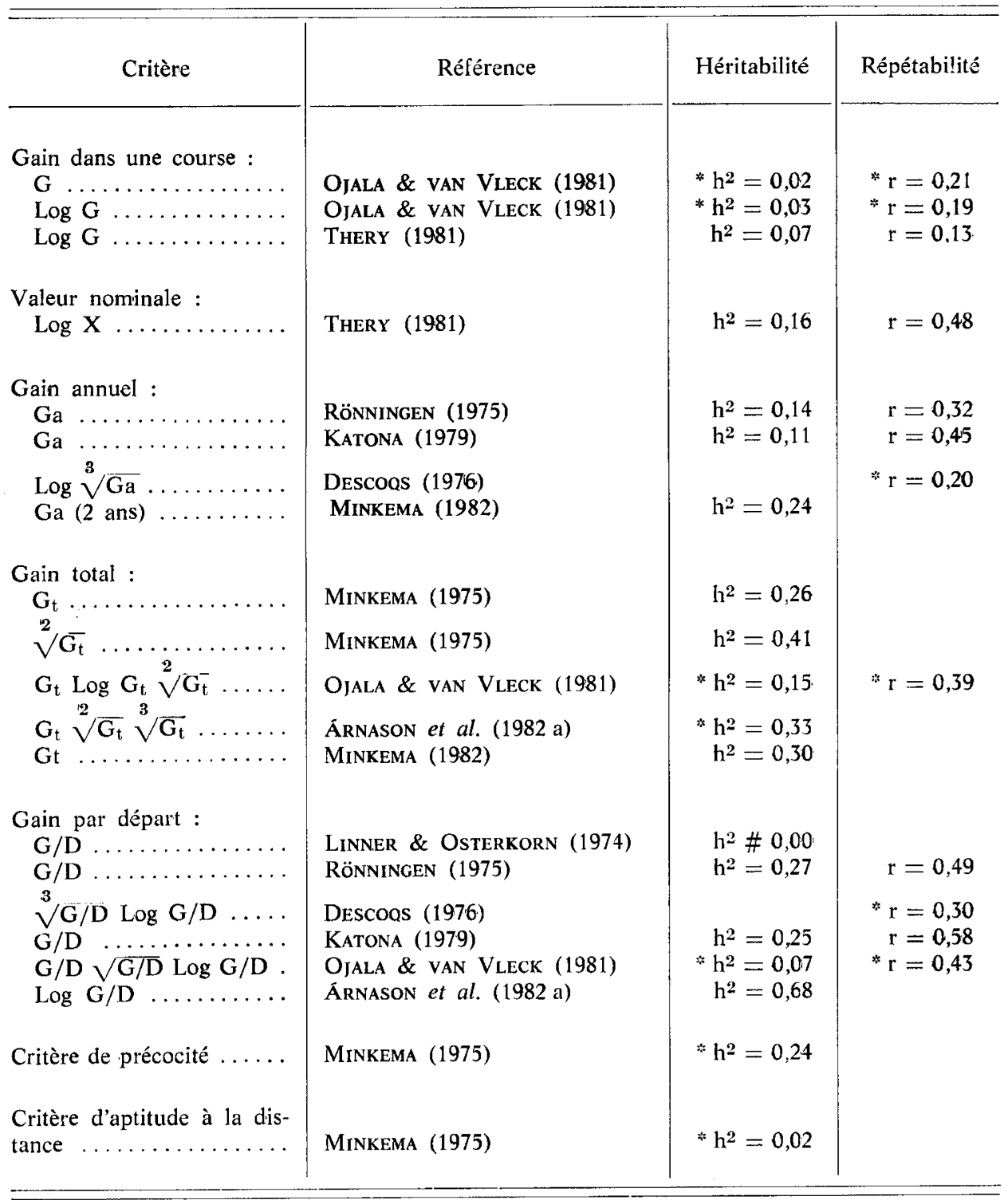


correspondants de Katona (1979) sont un peu plus faibles $(0,55$ à 0,76$)$. Cela traduit une assez bonne répétabilité des moyennes annuelles de temps qui s'accroît de 2 à 5 ans à mesure que les chevaux atteignent leur maturité.

\section{TABLEAU 3}

Récapitulatif des estimations de l'héritabilité et de la répétabilité des critères issus de la place. (les valeurs marquées d'un astérisque (*) sont des moyennes calculées par nos soins).

Heritability and repeatability of criteria resulting from the rank in a race.

(Values with an asterisk (*) are calculated means).

\begin{tabular}{|c|c|c|c|}
\hline Critère & Référence & Héritabilité & Répétabilité \\
\hline Rang d'arrivée & $\begin{array}{l}\text { OJALA \& VAN VLECK (1981) } \\
\text { THERY (1981) }\end{array}$ & $\begin{aligned} * \mathrm{~h}^{2} & =0,03 \\
\mathrm{~h}^{2} & =0,02\end{aligned}$ & $\begin{aligned} r & =0,21 \\
r & =0,05\end{aligned}$ \\
\hline Nombre de courses courues & ÁRNASON et al. (1982) & ${ }^{*} \mathrm{~h}^{2}=0,09$ & \\
\hline Nombre de $1^{\text {res }}$ places $\ldots$ & $\begin{array}{l}\text { RöNNINGEN (1975) } \\
\text { OJALA \& VAN VLECK (1981) } \\
\text { ÁRNASON et al. (1982) }\end{array}$ & $\begin{aligned} \mathrm{h}^{2} & =0,19 \\
* \mathrm{~h}^{2} & =0,11 \\
\mathrm{~h}^{2} & =0,22\end{aligned}$ & $\begin{array}{l}r=0,33 \\
r=0,34\end{array}$ \\
\hline Nombre de fois non placé & RöNNINGEN (1975) & $\mathrm{h}^{2}=0,23$ & $r=0,36$ \\
\hline Nombre de fois placé .... & $\begin{array}{l}\text { OJALA \& VAN VLECK (1981) } \\
\text { ÁRNASON et al. (1982) }\end{array}$ & $\begin{aligned} * h^{2} & =0,18 \\
h^{2} & =0,26\end{aligned}$ & $* r=0,33$ \\
\hline Régularité de l'allure .... & $\begin{array}{l}\text { RöNNiNGEN (1975) } \\
\text { KATONA (1979) } \\
\text { OJALA \& VAN VLECK (1981) }\end{array}$ & $\begin{array}{r}\mathbf{h}^{2}=0,07 \\
\mathrm{~h}^{2}=0,17 \\
\mathrm{~h}^{2}=0,08\end{array}$ & $\begin{aligned} r & =0,33 \\
r & =0,25 \\
r & =0,25\end{aligned}$ \\
\hline $\begin{array}{l}\text { Distance des courses où le } \\
\text { cheval a été p'acé } \ldots . . .\end{array}$ & THERY (1981) & $h^{2}=0,06$ & $r=0,12$ \\
\hline
\end{tabular}

Les chiffres correspondants pour le temps record sont moins élevés mais tout à fait comparables : 0,44 à 0,64 pour KATONA (1979) et 0,34 à 0,62 pour Descops (1976) et 0,49 à 0,74 de 4 à 10 ans pour Ojala (1982). Ceci traduit la moins bonne qualité de l'information apportée sur un cheval par le record annuel par rapport à la moyenne des temps. La corrélation entre ces deux types de critères reste cependant élevée au niveau de l'année $(0,85)$ et ne s'abaisse que modérément $(0,68)$ au niveau de l'ensemble de la carrière selon Katona (1979) du fait essentiellement de l'absence de correction des records pour l'effet important de l'âge. Il en résulte que le record absolu d'un cheval ne représente le plus souvent que le meilleur temps de sa dernière année de compétitions. C'est ce que HINTZ (1980) souligne à propos du trotteur américain en constatant que 78 p. 100 des temps records du Stud Book théoriquement obtenus entre 2 et 12 ans l'étaient en fait à 3 et 4 ans, période où justement la carrière 
s'arrête le plus fréquemment. Ceci pourrait expliquer le fait que le temps record absolu de toute la carrière n'apporte, semble-t-il, pas beaucoup plus d'information qu'un temps record annuel ou une moyenne annuelle.

Les études sur les critères autres que le temps sont à la fois moins nombreuses et plus disparates (tabl. 2 et 3 ). Il apparaît néanmoins que dans certains cas ce type de mesure des performances peut conduire à d'utiles prolongements pour la sélection, les valeurs d'héritabilité trouvées (MINKEMA, 1975 ; ÁRNASON et al., 1982 a) étant parfois sensiblement plus élevées que celles fournies par les valeurs chronométriques.

Cela n'apparaît cependant pas d'une façon générale (LinNer \& Osterkorn, 1974 ; Ojala \& VAN Vleck, 1981 ; Thery, 1981) et nous allons donc essayer d'analyser ces résultats d'une façon plus approfondie.

Le gain dans une course se déduit du classement et de la dotation de l'épreuve par des règles strictes qui sont de type exponentiel (THERY, 1981). Or, il apparaît que le rang d'arrivée est à la fois très peu héritable et très peu répétable. Cela se traduit par une faible héritabilité du gain dans une course qui, malgré les précautions prises au niveau des distributions par l'utilisation de transformations, demeure inférieure à 10 p. 100. La valeur nominale des prix dans lesquels un cheval s'est classé traduit le niveau des courses sans prendre en compte les classements : elle se trouve de ce fait sensiblement plus héritable et plus répétable.

Quand on cumule les résultats au niveau de l'année ou de la carrière, il apparaît que le nombre de courses disputées par le cheval est un caractère peu héritable (ÁRNASON et al., 1982 a). En revanche, le nombre de fois où il a été classé $1^{\mathrm{er}}, 2^{\mathrm{e}}$, $3^{\mathrm{e}}, 4^{\mathrm{e}}$, a une héritabilité voisine de 0,20 qui n'est pas négligeable. Cela se traduit pour les gains annuels ou les gains totaux par des valeurs d'héritabilité également non négligeables. Le spectre des estimations est cependant beaucoup plus étendu que pour les chronométrages et illustre les problèmes statistiques particuliers du traitement de cette variable et le fait qu'un gain traduit essentiellement un programme de courses qui peut être très différent d'un pays à l'autre.

On peut en effet imaginer deux modalités extrêmes de réalisation d'un gain total. L'une suppose un programme de courses peu hiérarchisé ; le gain total dépendra alors du nombre de fois où le cheval s'est classé qui devient alors un critère héritable. Le nombre de départs est fortement influencé par l'entraîneur, les courses ouvertes au cheval étant peu limitées. Son héritabilité reste donc faible. Cela a pour conséquence d'introduire dans le gain moyen par départ une variabilité due aux effets du milieu qui diminue son héritabilité par rapport au gain total (OJALA \& VAN VLECK, 1981). A l'opposé, l'autre modalité suppose un programme très hiérarchisé qui n'offre qu'une gamme limitée de courses à un cheval en fonction des gains déjà acquis. Le nombre des courses courues par le cheval ne dépend plus alors autant de l'entraîneur et le gain moyen par départ rend alors mieux compte du niveau moyen des courses dans lesquelles le cheval se place. L'effet des classements se trouve alors en partie tamponné et l'on aboutit à une situation inverse de la précédente, le gain moyen par départ devenant plus héritable que le gain (ÁrNASON et al., $1982 \mathrm{a}$ ).

Le fait marquant de l'étude de ces critères notamment en France (THerY, 1981), est la mise en évidence de la prépondérance dans l'évaluation des performances en course du niveau de l'épreuve disputée par rapport au résultat obtenu dans cette même épreuve. La variation entre courses des critères de performances prime très largement 
sur la variation intra-course. Pour les variables de gains, cette primauté est d'autant plus forte que le programme est hiérarchisé et que la distribution des valeurs nominales de course est étalée mais il faut souligner qu'elle est également caractéristique des valeurs chronométrées où les variations intra-course de quelques dizièmes de seconde sont négligeables devant les variations entre courses qui sont de plusieurs secondes.

Ceci nous amène à discuter de l'intérêt relatif des valeurs chronométrées et des gains pour conduire la sélection. Nous avons vu que, pour utiliser les premiers, il fallait corriger pour de nombreux effets systématiques au premier rang desquels se situent l'âge, l'hippodrome, le type de départ, le mode attelé ou monté de la course, la distance... Cela ne pose en théorie pas de difficultés statistiques particulières si ce n'est la gcution de fichiers très volumineux qui est souvent un obstacle important. On voit donc se développcr le recours à des critères synthétiques au premier rang desquels se situe le temps rccord. Largumentation développée pour justifier son utilisation suppose a priori qu'au cours de sa carrière, tout cheval a la possibilité d'exprimer le meilleur de lui-même dans les meilleures conditions. Retenir la meilleure performance «dispenserait» alors de corriger pour les effets du milieu. Cette proposition, comrice en témoignent les mentions qui accompagnent les publications des records c'anj les Stud-Books, n'est sans doute qu'en partie vérifiée. En effet, le temps record apparaît à la suite de ces travaux comme un temps parmi les autres, ne fournissant guère plus d'information qu'un autre. Simplement, c'est celui qui sanctionne la participation du cheval à la course la plus rapide de sa carrière.

En ce sens, si l'on prend comme estimateur de la valeur d'un cheval la plus forte valeur nominale des courses dans lesquelles ce cheval a été classé, cela n'apparaît pa; fondamentalement différent. A notre sens, il ne faut donc pas systématiquenent opposer les valeurs chronométrées aux autres critères qui ne font que mesurer de façon différente le même phénomène. Simplement dans un cas le niveau de l'épreuve est estimé par un chronométrage, dans l'autre par une dotation.

On pelit par ailleurs souligner que, si les chevaux courent rarement pour battre un record, ils courent en revanche très souvent pour obtenir des gains. Il y a donc dans ce dernier critère des possibilités de mesure des performances qui dépassent peut-仓̂tre celles fournies par les chronométrages dont l'héritabilité reste moyenne. Leur inconvénient majeur est qu'ils sont, en raison de leur métrique, beaucoup plus difficiles à traiter correctement du point de vue statistique. Cela nécessite le recours à des transformations qui ne sont pas toujours parfaitement opérantes. En revanche, les corrections des effets systématiques du milieu sont en général plus simples car il s'agit essentiellement de l'année de la performance, de l'âge et du sexe. Un autre avantage de ces critères est qu'ils concernent en général une proportion plus importante des animaux, bien que cela ne semble pas vérifié dans tous les pays (OJala \& VAN VLECK, 1981).

Pour toutes ces raisons, les valeurs d'héritabilité des gains ont été trouvées dans la littérature à la fois comme étant les plus fortes (Minkema, 1975 ; ÁrNason et al., 1982 a), mais également les plus faibles (Linner \& Osterkorn, 1974 ; OJALa \& van VLECK, 1981).

Signalons également d'autres caractéristiques intéressantes du trotteur qui n'ont pas été mesurées par le chronométrage. Il s'agit de : 
- la régularité des allures dont l'héritabilité voisine de 0,10 apparaît peu élevée (Rönningen, 1975 ; Katona, 1979 ; Ojala \& Van Vleck, 1981),

- la précocité dont 20 à 25 p. 100 de la variation peut être considérée comme d'origine génétique (Minkema, $1975 \mathrm{a}$ ),

- et de l'aptitude à la distance qui, chez le trotteur, n'apparaît ni héritable ni répétable (Minkema, 1975 ; Thery, 1981).

\section{Estimation de la valeur génétique des reproducteurs}

Le progrès génétique annuel est proportionnel à la précision de l'estimation de la valeur génétique, à l'intensité de la sélect:on et à la brièveté de l'intervalle entre générations.

Le choix des reproducteurs d'après leurs propres performances est donc la méthode d'amélioration génétique la plus efficace compte tenu des valeurs d'héritabilité citées plus haut. On peut de cette façon ne retenir que 2 à 3 p. 100 des mâles et sans doute moins de 50 p. 100 des pouliches (de Richter, 1977). Ce schéma est à peu près réalisé dans la pratique pour la sélection des étalons. Pour le choix des poulinières en revanche, il est appliqué d'une manière beaucoup plus approximative.

Le critère utilisé pour conduire cette sélection et qui sert dans la réglementation est le temps record dont l'héritabilité paraît satisfaisante. Les auteurs allemands lui préfèrent cependant la moyenne des temps qui leur paraît, en fonction de son déterminisme génétique et de lintensité de sélection réalisable, être le critère susceptible de conduire aux meilleurs résultats.

La même démarche amène RöNNINGEN (1975) et Minkema (1976) à préconiser les gains qui permettent de réaliser de plus forts taux de sélection tout en ayant des valeurs d'héritabilité comparables, sinon meilleures. La recherche d'une augmentation de la précision du critère de sélection peut également conduire à associer deux ou plusieurs variables supposées mesurer la même chose (ÁRNASON et al., 1982 a). On pourrait ainsi à titre d'exemple associer «gains » et « record » dans un même critère de choix. Cela suppose cependant une bonne connaissance de la corrélation génétique des variables que l'on désire associer et de travailler sur des distributions bien normalisées. A titre d'exemple, la corrélation génétique entre le gain et le record (variables transformées) a été trouvée proche de 1 par Minkema (1975) alors que les valeurs trouvées par RönNiNGEN (1975) sur des variables non transformées ne sont que de l'ordre de 0,50 . Il ressort néanmoins que le contrôle des performances a une précision qui demeure toujours relativement faible quelles que soient les améliorations susceptibles de lui être apportées.

Pour avoir une précision supérieure il faut procéder à un contrôle de la descendance. En effet, bien que cela rallonge l'intervalle de génération, Ström \& PhilipsSON (1978) ont montré qu'en association avec le contrôle individuel il peut améliorer le progrès génétique annuel de 2 à 17 p. 100. Ce test de la descendance est d'ailleurs pratiqué «à l'estime » par les éleveurs comme le montre l'utilisation très différentielle des étalons. 
La méthode chiffrée la plus simple mais aussi la plus grossière consiste à calculer le pourcentage des descendants d'un étalon ayant atteint un certain seuil de performances. C'est ainsi qu'on a pu entendre parler du pourcentage de chevaux qualifiés ou du pourcentage de chevaux ayant couru en moins de ..., etc. Cette méthode est d'ailleurs utilisée dans certains pays de 1Est (Fomin, 1972; Suciu \& Dobrescu, 1970). Outre l'imprécision d'un pourcentage calculé sur un fabile nombre de descendants, elle ne tient compte d'aucun effet du milieu (année, âge, ...) et n'apparaît donc pas recommandable.

Les autres techniques proposées retracent l'évolution de la méthodologie de l'estimation de la valeur génétique des taureaux laitiers. On retrouve (ÓCSAG, 1963) l'utilisation de la comparaison mère descendant intra père sur des effectifs encore plus limités que chez les bovins.

Ce procédé est ensuite remplacé par la classique comparaison aux contemporains avec correction pour la qualité des mères chez certains auteurs (MINKEMA, 1976; ÁRNASON et al., 1982 a) et sans correction pour les autres. Enfin, les généticiens tentent actuellement de mettre en wuvre les estimations du BLUP (Best Linear Unbiased Predictor) qui constituent en la matière le «dernier cri » de la technique (VAN VlecK \& Hintz, 1975 ; Distl et al., 1982 ; ÁrNASON, 1982 ; ÁrNASON et al., 1982 b). Cela pose toutefois un certain nombre de problèmes de résolution des calculs numériques qui nécessitent une haute spécialisation à la fois en informatique et en statistique.

\section{Conclusion}

Les recherches en génétique quantitative sur le trotteur ont connu ces dernières années un brusque développement. Les performances en course mesurées par le chronomètre ont une héritabilité moyenne comprise entre 0,20 et 0,30 : si cette valeur justifie la sélection d'après les performances propres, elle ne permet toutefois pas d'exclure un contrôle de la descendance des étalons qui est le seul à pouvoir fournir des estimations précises de leur valeur génétique.

A l'avenir, l'analyse approfondie d'autres variables comme les gains devrait permettre soit de dégager des critères plus héritables que le chronomètre, soit d'adjoindre cette information aux réductions kilométriques pour gagner en précision. Cela nécessite cependant un effort de recherche important déjà entamé dans plusieurs pays et notamment en France.

Dans la pratique, le passage de l'évaluation des reproducteurs par leur temps record absolu ou ceux de leurs descendants à des indices plus sophistiqués sur le plan statistique et génétique et incontestablement plus précis devrait aider les éleveurs. Toutefois, la participation des organisations professionnelles est absolument indispensable à l'élaboration de tels projets si l'on ne veut pas que le débat reste cantonné à quelques universités américaines et européennes et le fait de quelques spécialistes seulement. 


\section{Summary \\ Heritability of Trotting performance - $A$ bibliographical review}

Litera:ure on quantitative genetics of trotters shows that the heritability of Trotting performance has a medium value $\left(0.20<\mathrm{h}^{2}<0.30\right)$.

The criteria available from race results express the level of the races in which a horse may run rather than its real theoretical potential. From that point of view there is little difference between timing and earning criteria. In one case, the level of the race is expressed by the mean speed of the race, in the other one by the amount of money distributed in the race.

The incidences of systematical effects of age, sex, year of performance, racetrack, autostart... are reviewed as well as statistical difficulties connected with the particular distributions of other variables than timings.

Estimation of the genetic value of a breeding animal is therafter analysed in terms of performance and progeny tests.

The possible development of research in that field and its applications, are discussed.

\section{Références bibliographiques}

ÁrNAson T., 1982. Prediction of breeding values for multiple traits in small non-random mating (horse) populations. Acta Agriculturae Scandinavica, 32, 171-176.

Árnason T., Darenius A., Philipsson J., 1982 a. Genetic selection indices for swedish trotter broodmares. Livest. Prod. Sci., 8, 557-565.

Árnason T., Darenius A., Bendroth M., Fhilipson J., 1982 b. Estimation of breeding values in horses, with an example of use in swedish trotters. Journées d'études de la commission chevaline de la F.E.Z., Leningrad, 16-19 août 1982.

Csapó G., 1968. Tableau mensuel des temps moyens au trot à différents âges durant l'année. Kisérl. Közl. Allaten., 61 B, 31-50.

Descops C., 1976. Recherche de critères d'estimation du potentiel d'un trotteur français à partir de ses performances en course. Mémoire de fin d'études, I.N.A., Paris. Polycop., C.N.R.Z., 62 p.

Distl O., Katona Ö., KräUsslich H., 1982. Vergleich der Zuchtwertschätzmethoden BLUP and $\mathrm{CC}$ beim Traber. Züchtungskunde, 54, 157-164.

DušEK J., 1979. Abschätzung der Heritabilitätskoeffizienten der Geschwindigkeit von Trabern der ungarischen und italienischen Zucht. Die Bodenkultur, 30, 79-84.

Ferguson P.W., Harvey W.R., 1982. Effect of the speed of the winner on time for individual horses to trot or pace a mile. Abstr. $\mathrm{n}^{\circ}$ 65, Annual Meet. Am. Soc. Anim. Sci. Can. Soc. Anim. Sci., Guelph, 8-11 août 1982.

Fomin A.B., 1972. Efficacité de différentes méthodes de sélection chez le trotteur. Doklady T.S.Kh.A., 185, 129-132.

Galton F., 1898. An examination into the Registered speeds of American Trotting Horses, with remarks to their value as Hereditary Data. Proc. Roy. Soc., 62, 310-315 (Cité d'après MinKema, $1975 \mathrm{a}$ ).

Georgescu G., Curelariu N., Stoica A., 1979. Etude de quelques paramètres génétiques $\mathrm{du}$ trotteur roumain. Lucrari Stiintifice Institutul Agronomic «N Balescu» D. Zootechnie, 20-21, 139-143.

GopKA B.M., 1971 a. Héritabilité de la vitesse chez le trotteur Orlov. Naukovi Pratsi U.S.G.A. n 41, 169-171 (Cité d'après Anim. breed. Abstr., 40, n 5 139). 
GopKa B.M., 1971 b. Héritabilité de la vitesse chez le trotteur Orlov. In : Genetika i seleksiya na Ukraine ch. 2 Kiev : Nauk. Dumka P. 8 (Cité d'après Anim. breed. Abstr., 40, $\left.\mathrm{n}^{\circ} 1334\right)$.

Gopka B.M., Derev'yanchuK A.V., 1970. Héritabilité de la vitesse chez les trotteurs. Doslid. Tvarin. Kiïv, $\mathrm{n}^{\circ}$ 38, 100-102 (Cité d'après Anim. breed. Abstr., 40, $\mathrm{n}^{\circ} 1335$ ).

Hintz R.L., 1980. Genetics of performance in the horse. J. Anim. Sci., 51, 582-594.

HiNTZ R.L., VAN VLeCK L.D., 1978. Factors influencing racing performance of the standard bred pacer. J. Anim. Sci., 46, 60-67.

KalmYKov A.N., 1973 a. Héritabilité de quelques caratères d'importance économique chez le trotteur Orlov. Genetika, 9, 50-58.

KaLmYKov A.N., 1973 b. Utilisation de l'héritabilité dans la sélection des trotteurs. Konevodstvo $i$ Konnyi Sport, $\mathrm{n}^{\circ} 8,16-17$.

KaLmYKov A.N., 1975. Héritabilité de caractères quantitatifs chez le trotteur. Uchenye Zapiski Kasanskogo Gosudartsvennogo Veterinarnogo Institutu, $\mathrm{n}^{\circ}$ 121, 23-27 (Cité d'après Anim. breed. Abstr., 46, n 3156 ).

Katona Ö., 1979. Genetical statistical analysis of traits in the german trotter. Livest. Prod. Sci., 6, 407-412.

Katona Ö., Osterkorn K., 1977. Genetisch - Statistische Auswertung des leistungsmerkmals Rennzeit in der deutschen Traberpopulation. Züchtungskunde, 49, 185-192.

LANGLoIs B., 1982. Quelques réflexions au sujet de l'utilisation du gain pour apprécier les performances des chevaux trotteurs. Polycop. C.N.R.Z., Jouy-en-Josas, France, 16 p. et 3 annexes.

LinNer M.Th., OSTERKoRN K., 1974. Züchterische Auswertung der Rennleistung von Traberpferden der Jahrgänge 1963 and 1964 in der B.R.D. Züchtungskunde, 46, 168-176.

LonkA T., 1946. The evaluation of the pulling ability of horses. Valt. maatal. Koetoim. Julk., 126, 1-50 (Cité d'après VARO, 1965).

Minkema D., $1975 \mathrm{a}$. Studies on the genetics of trotting performance in Dutch Trotters : I: The heritability of trotting performance. Ann. Génét. Sél. Anim., 7, 99-121.

Minkema D., 1975 b. Genetic studies on performance in dutch trotters. Proc. Internat. Symp. Genetics Horse-Breed., Dublin, 17-18 sept. 1975, 24-32.

Minkema D., 1976. Studies on the genetics of trotting performance in Dutch trotters : II. A method for the breeding value estimation of trotter stallions. Ann. Génét. Sél. Anim., 8, 511-526.

Minkema D., 1978. Leistungsprüfung und Zuchtplanung in der Traberzucht (Stand des Wissens). Journ. ét. Comm. Chev. Féd. Europ. Zootech., Stockholm, 5-7 juin 1978.

Minkema D., 1981. Studies on the genetics of trotting performance in Dutch trotters : III - Estimation of genetic change in speed. Ann. Génét. Sél. Anim., 13, 245-254. Minkema D., 1982. An analysis of earnings of Dutch trotters born between 1959 and 1971. Rapport B-197, juin 1982, I.V.O. "Schoonoord», Zeist, Hollande, 22 p.

OCSAG I., 1963. Welche methoden eignen sich zur Beurteilung des Schnelligkeitsvererbungsvermögens der rennpferdzuchthengste. Acta. Agron. Hung. (Budapest), 12, 181-207.

OCSAG I., Tóth I., 1959. Héritabilité de la vitesse chez les chevaux. Agrartud. Egyet Mezögtud. Kar. Közl., Gödöllö, 61-67.

Ojala M.L., 1982. Some parameters estimated from a restricted set of race records in trotters. Acta Agriculturae Scandinavica, 32, 215-224.

OJala M.J., VAN Vleck L.D., 1981. Measures of race track performance with regard to breeding evaluation of trotters. J. Anim. Sci., 53, 611-619.

DE Richter A., 1977. Estimation de quelques paramètres démographiques du trotteur français. C.E.Z. Rambouillet, rapport de stage, 35 p.

RöNNINGEN K., 1975. Genetic and environmental factors for traits in the North-Swedish trotter. Z. Tierz. Züchtbiol., 92, 164-175.

SChWARK H.J., Freund H., 1974. Zur Problematik der Zuchtwertanalyse bei der Züchtung von Rennpferden. I. Intern. Wissensch. Sympos., 124-143, Leipzig, 1974. 
Schwark H.J. ,FreUnd H., 1976. Zuchtwirksame Kriterien im Leistungsprüfungssystem von Trabrennpferden. II. Intern. Wissensch. Sympos., 155-161, Leipzig, 1976.

STröm H., Philipsson J., 1978. The relative importance of performance tests and progeny test in horse breeding. Livest. Prod. Sci., 5, 303-312.

Suciu T., Dobrescu O., 1970. Contribution à l'amélioration du contrôle de la descendance des étalons trotteurs. Lucr. Stiint. Inst. Cerc. Zootech., 27, 447-454.

THERY C., 1981. Analyse génétique et statistique des performances des trotteurs français en course en 1979 et 1980 . Mémoire du D.E.A. de génétique quantitative et appliquée, Paris $\mathrm{XI}^{\mathrm{e}}$, polyp., C.N.R.Z., 83 p.

Tolley E.A., NotTer D.R., 1982. Heritability and repeatability of racing performance for standardbred horses. Abstr. $n^{\circ} 120$, Annual Meet. Am. Soc. Anim. Sci. Can. Soc. Anim. Sci., Guelph, 8-11 août 1982.

Vainikainen V., 1946. Breeding the Finnish Horse. Suomen maatal. Tiet Seuran Julk., 62, 1-117 (Cité d'après VARO, 1965).

VAN Vleck L.D., Hintz R.L., 1975. Prediction of genetic value of stallions. Proc. Internat. Symp. Genetics Horse-Breed., Dublin, 17-18 sept. 1975.

Varo M., 1965. Some coefficients of heritability in horses. Annales Agriculturae Fenniae, 4, 223-237.

WrankmoRe F., 1976. Rennleistung und exterieurmerkmale beim Traber. II. Intern. Wissensch. Sympos., 162-170, Leipzig, 1976. 